GUANGZHI ZANG, Ph.D. ${ }^{1}$

E-mail: gzzang@bjtu.edu.cn

MENG XU, Ph.D. ${ }^{1}$

(Corresponding author)

E-mail: mengxu@bjtu.edu.cn

ZIYOU GAO, Ph.D. ${ }^{1}$

E-mail: zygao@bjtu.edu.cn

${ }^{1}$ State Key Laboratory of Rail Traffic Control and Safety

Beijing Jiaotong University, Beijing 100044, P.R. China
Traffic Management Original Scientific Paper Submitted: 31 Oct. 2016 Accepted: 10 Oct. 2017

\title{
HIGH-OCCUPANCY VEHICLE LANES AND TRADABLE CREDITS SCHEME FOR TRAFFIC CONGESTION MANAGEMENT A BILEVEL PROGRAMMING APPROACH
}

\begin{abstract}
High-occupancy vehicle (HOV) lanes, which are designed so as to encourage more people to use high-capacity travel modes and thus move more people in a single roadway lane, have been implemented as a lane management measure to deal with the growing traffic congestion in practice. However, the implementation has shown that some HOV lanes are not able to achieve the expected effects without proper HOV lane settings. In this study, the tradable credits scheme (TCS) is introduced to improve the HOV lane management and an optimal capacity of HOV lanes in a multilane highway is investigated to match TCSs. To approach the investigation, a bilevel programming model is proposed. The upper-level represents the decision of the highway authority and the lower-level follows the commuters' user equilibrium with deterministic demand. The potential influence of TCSs is further investigated within the proposed framework. A modified genetic algorithm is proposed to solve the bilevel programming model. Numerical examples demonstrate that combining TCSs with the HOV lane management can obviously mitigate traffic congestion.
\end{abstract}

\section{KEY WORDS}

high-occupancy vehicle lanes; lane management; tradable credits scheme; travel demand management; equilibrium;

\section{INTRODUCTION}

Transport plays a pivotal role in the nation's economy and quality of life. However, in many cities, road transport as a public entity suffers from over-consumption due to lack of clear property rights and results in traffic congestion. Road traffic congestion has a range of undesirable consequences, which includes negative economic impacts resulting from the inefficient and unreliable distribution and delivery of goods, services, and resources, lost productivity, increased vehicle operating costs, and environmental pollution.

The concept 'managed lanes' covers a variety of strategies and techniques. Many of them have been used for years in traffic congestion management. The fundamental rationale behind the managed lanes is to employ operational tools to maximize the productivity of the available roadway [1]. There are different lane management measures, e.g., high-occupancy vehicle (HOV) lanes, which encourage more people to use high capacity travel modes, and thus move more people on a single roadway lane. High-occupancy toll (HOT) lanes, which allow vehicles to purchase access (through tolls) to use underutilized HOV lane capacity, thus maximizing vehicle use of HOV lanes without sacrificing HOV speed and reliability, and providing revenue to help pay for HOV lane construction, maintenance and operation.

HOV lanes have been implemented as a form of managed lane measure to deal with the growing traffic congestion in practice. However, they are probably facing different cases in practice, i.e. besides some underutilized HOV lanes sometimes, there are also some HOV lanes being heavily used, especially during peak periods. In 2016, Shenzhen municipal opened the first HOV lane in China, which was set in the inner lane of the Binhai Avenue from west to east with the service time from 7:30 a.m. to 9:30 a.m. and 5:30 p.m. to 7:30 p.m. The opening of HOV lane brings burden to monitoring and management, and also, the attitude of the public to the implementation of HOV lane is doubtable [2]. Shewmake [3] reviewed studies on the impacts of HOV lanes with the focus on behavioural models, and emphasized that despite many years of building HOV lanes, it is still unclear whether they increase the welfare, reduce congestion and vehicle miles travelled (VMT), or improve air quality. HOT has been implemented in the USA on several interstate highways, although it still faces several challenges, e.g., toll designing, public resistance to HOV/HOT changes. Similarly, there are successful cases for congestion pricing, e.g., in London, Singapore, and Stockholm. However, there is no hesitation that social and political resistance to road congestion pricing is still strong. 
There are some studies discussing HOV lanes and HOT lanes from the overview of social welfare maximization with a static equilibrium model approach. Yang [4] evaluated the efficiency of HOV lanes through cost-effectiveness analysis using a simple modal choice equilibrium model. Yang and Huang [5] presented a theoretical model to deal with carpooling behaviour and optimal congestion pricing in a multi-lane highway with or without HOV lanes. Konishi and Mun [6] presented a detailed theoretical analysis of a simple model of commuters using a highway with multiple lanes including HOV lanes or HOT lanes.

Related studies also include some theoretical analysis of two parallel routes. Small and Yan [7] investigated "product differentiation" of two parallel routes. They used a model with two groups with a different value of time (VOT) to investigate relative performance of social welfare. Moreover, Verhoef and Small [8] proposed a static equilibrium model of two parallel routes on which commuters are continuous heterogeneous on VOT. For an overview of the development of HOV facilities, see Fuhs and Obenberger [9], and on related ridesharing systems, see Furuhata et al. [10], who provide a classification that reveals issues with the state-of-the-art ridesharing systems. In the section of "Institutional design" in Fuhs and Obenberger [9], they reviewed lane management measures with respect to HOV lanes, HOT lanes and other special types of managed lanes. The existing studies provide multiple views of the HOV lane management. However, the fundamental issue for HOV lanes, how to set HOV lanes and determine the optimal capacity of HOV lanes when the total capacity of the highway remains unchanged, has not been sufficiently examined yet and has been kept open.

Furthermore, de Corla-Souza [11] proposed the concept of FAIR highways which is an extension of HOV-related lanes. A FAIR highway system is a highway management system which converts all lanes of the highway into HOT lanes and urges low-occupancy vehicles (LOVs) that would not like to pay the toll to choose other alternative roads during the peak hours. Meanwhile, these tolls will be refunded to low-income commuters who may be negatively affected by the toll highway. Fan et al. [12] presented a hypothetical FAIR highway corridor under a bilevel programming framework.

An alternative way is to consider managing mobility on HOV lanes with a tradable credits scheme (TCS), which provides a new approach for the mobility management of carpooling commuters. A TCS, which covers a variety of instruments that range from the introduction of flexibility into regulation to the organization of competitive markets with credits, has received much attention for traffic congestion management [13]. A TCS can reward sustainable travel patterns and provide a continuing incentive for travellers to managing their credits, e.g., reducing credit use by carpooling or using public transportation. Through effective design with credits, it is possible to achieve desirable outcomes such as income redistribution, transfer of credits between different groups, and improvement of using HOV lanes.

The TCS measure has become familiar to environmental economists as a pollution control measure. This is in contrast to the case for many transport economists and transport management practitioners, for whom the TCS measure is still a research topic for mobility management. Researchers in transport economics can realize the potential of a TCS for mobility management, although it is clear that many theoretical and application-related issues remain undeveloped. Recently, Yang and Wang [14] investigated how to obtain the equilibrium traffic flow pattern and the credit market price in the fixed demand case for a given credits scheme. For a recent overview on TCSs, Fan and Jang [15] reviewed a variety of TCSs for roadway capacity allocation focusing on detailed system design and overall functions.

This paper deals with the HOV lane management problem in a multi-lane highway. In reality, cooperating with other management measures of HOV lanes, the HOT policy is a flexible and effective measure which enables the improvement of the utilization of HOV lanes by allowing LOVs that pay toll to use HOV lanes. However, it concerns the congestion pricing which can lead to public protests. In this respect, the TCS policy has its natural advantage for its fiscal neutrality. The TCS allows commuters to obtain credits in two ways, freely and equally distributed by a highway authority or purchased on an open market from other commuters. Under these circumstances, if commuters want to choose a particular travel mode and do not have enough credits, they are forced to spend a certain amount of money to purchase them at a reasonable price.

This paper concentrates on two main research questions. (1) Is the TCS policy able to reduce the total commuting time with the presence of HOV lanes? (2) How to calculate the optimal capacity of HOV lanes and the optimal TCSs? The main contributions of this study are listed as follows.

1) A bilevel programming approach is presented to investigate the HOV lane management problem. The upper-level programming represents the decision of the highway authority and the lower-level programming follows the commuters' user equilibrium with deterministic demand [16]. Two Bilevel programming models are proposed to approach the problem with/without TCS, respectively. In order to introduce the influence of TCS, a linear constraint is added to the classical user equilibrium in 
the lower-level programming of the model with TCS. The optimal HOV lanes and TCSs setting are determined by the upper-level programming.

2) A modified genetic algorithm (GA) is proposed to solve the bilevel programming model with TCS. One-to-one mapping is designed to convert the values from the decision variables of the programming model to standard continuous chromosomes. Classical mutation, crossover, and selection processes are adopted in the algorithm. Numerical examples show the effectiveness and stability of the proposed algorithm. Besides, the model without TCS can also be solved by a simplified version of the proposed algorithm

3) Numerical examples indicate the functional principle of TCS. The main purpose of charging credits is to restrict a certain number of LOVs comparing to the equilibrium state. If this number is proper, the reduction of LOVs and increment of HOVs will lead to a reduction of the commuting time on GP lanes and a slight increase of the commuting time on HOV lanes, respectively. Ultimately, the total commuting time of all commuters will decrease. Furthermore, parameter analyses explain the impact on the result of two relative parameters.

This paper is organized as follows. In Section 2 the two bilevel programming models are proposed to describe the HOV lane management problem with/ without TCS and in Section 3 a modified GA for solving the proposed models is presented. Numerical experiments are presented in Section 4 and the paper is concluded in Section 5.

\section{PROBLEM FORMULATION}

\subsection{Description}

Assume that a fixed number of commuters need to drive to work on a multi-lane highway which connects a residential area and a work zone. Two travel modes are considered in this study, i.e. driving alone or forming a two-person carpool (the proposed model can easily extend to three or more persons). Commuters freely choose their travel modes. If they choose to drive alone, they are called LOV commuters. If they choose to form a carpool, they are called HOV commuters. The commuting time which is the commuting cost includes two parts, i.e. the travel time for both types of commuters and the carpool organization time only for HOV commuters. In our study, the carpool organization cost is assumed to be positive, since HOV commuters need to spend extra time to prepare for carpool in reality.

Table 1 gives the analysis on how LOV and HOV commuters choose types of lanes when they want to minimize their commuting cost. According to the HOV lane policy, LOV commuters do not have access to HOV lanes unlike HOV commuters who are entitled to use both GP lanes and HOV lanes. However, no HOV commuters will choose GP lanes, since the HOV commuters with the intention of using GP lanes will definitely drive alone and save the carpool organization cost. Therefore, all HOV commuters will choose HOV lanes in this study.

Table 1 - The choice rationale of LOV commuters and HOV commuters

\begin{tabular}{|l|c|c|}
\hline \hline & Using GP lanes & Using HOV lanes \\
\hline \hline LOV commuters & Allowed & Not allowed \\
\hline HOV commuters & $\begin{array}{c}\text { Allowed but no } \\
\text { one chooses }\end{array}$ & Allowed \\
\hline
\end{tabular}

The presence of LOV and HOV commuters makes the highway authority to decide at how much HOV lane capacity should be set in order to mitigate traffic congestion, which can be considered to optimize the highway from the supply side. Moreover, the highway authority has consideration for traffic congestion management from both supply and demand side, i.e., not only introducing HOV lanes (supply side) but also implementing TCS (demand side).

If TCSs are implemented, the commuting cost will include a new item, the credit cost, which means the cost one commuter spends or achieves by purchasing or selling credits. In particular, because VOT is assumed to be the same among all commuters, the credit cost is also measured in time units.

In this study, both cases, i.e. with or without TCSs, are investigated theoretically. If TCSs are implemented, the highway authority will equally distribute a certain number of credits to each commuter. Commuters choose driving alone or carpooling according to their willingness, and spend a different number of credits based on their choices. That is, all commuters who use the highway need to pay a certain number of credits; however, the charged number of credits for LOV commuters and HOV commuters are different. Those credits can be traded on an open market without transaction cost (see Remark 1 for more details) and the credits price is determined by the market only.

Remark 1. The transaction cost of credits cannot be avoided in the real case. In this study, since we focus on the influence of TCSs on commuters' behaviour, a relatively perfect market system of credits is assumed to be established by the traffic authority to reduce transaction cost as much as possible. Provided with this assumption, the transaction cost is neglected in the following models.

\subsection{Notation}

The number of all commuters $N$ is a given positive and exogenous parameter under the assumption of deterministic travel demand. The variable $n$ means the number of HOV commuters, and $N-n$ represents the 
number of LOV commuters. $C$ denotes the capacity of the highway. The travel times of LOV and HOV commuters are depicted by the travel time function $f_{T}$ which is a function of the number of commuters and capacity of corresponding type of lanes. The carpool organization cost $D$ is positive. The ratio of HOV capacity to total highway capacity or HOV capacity ratio for short $\rho$ restricted to the range $[0,1]$ can be considered as a comprehensive index (see Remark 2 for more details). Without loss of generality, it is assumed that the total number of distributed credits is $N$ (see Remark 3 for more details). Furthermore, every commuter who travels on this highway needs to pay a certain number of credits if TCS is implemented. The LOV credit toll $\kappa_{1}$ or the HOV credit toll $\kappa_{2}$ are the number of credits that one LOV commuter or one HOV commuter should be charged by the highway authority. Considering that HOV commuters consist of two persons per car, each $\mathrm{HOV}$, therefore, should be charged by $2 \kappa_{2}$.

Remark 2. The HOV capacity ratio is assumed to be a real number between 0 and 1 as a continuous decision variable in the following programming. This value takes into account not only the ratio between the number of HOV lanes and total lanes, but also some parameters of relevant facilities, such as road width and area of the entrance zone.

Remark 3. In the proposed model, it is assumed that the traffic authority distributes 1 credit to each commuter and hence the total number of credits is $N$. In practice, it will not change the cost and revenue of commuters, so long as the highway authority distributes the credits to all commuters equally and alters the credit tolls correspondingly. For example, if each commuter gets 1 credit and should be charged 1.5 credits, the highway authority can distribute 10 credits and charge 15 credits. This change will only lead to the price of credit being 1/10 than before, but the cost of buying enough credits is the same.

\subsection{HOV lane management without TCS}

The HOV lane management problem to determine the optimal HOV capacity ratio is approached by Bilevel Programming 1-4.

$$
\begin{aligned}
& \min _{\rho}\left[f_{T}\left(\frac{1}{2} n, \rho C\right)+D\right] n+ \\
& +f_{T}((N-n),(1-\rho) C)(N-n)
\end{aligned}
$$

subject to

$$
0 \leq \rho \leq 1
$$

where $n$ solves the following optimization problem

$$
\min _{n} \int_{0}^{n}\left[f_{T}\left(\frac{x}{2}, \rho C\right)+D\right] d x+\int_{0}^{N-n} f_{T}(x,(1-\rho) C) d x
$$

subject to

$$
0 \leq n \leq N
$$

The total commuting time which means the sum of all commuters' commuting time is formulated as Equation 1 which is also the objective function of the upper-level programming. The HOV capacity ratio $\rho$ is the decision variable restricted to the range $[0,1]$, as given by Constraint 2 . The objective function of the lower-level programming, Equation 3, conforms with the classic objective function of the user equilibrium and the Constraint 4 restricts the number of HOV commuters $n$ to $[0, N]$.

The bilevel programming provides a simple approach to the HOV lane settings under the consideration of travellers' equilibrium behaviour, that is, when the equilibrium state is achieved, no commuter can reduce their own commuting cost by changing their commuting modes (driving alone or carpooling). The decision variable of the upper-level programming is $\rho$, which determines how much highway resource will be used for HOV lanes.

\subsection{HOV lane management with TCS}

As mentioned, TCS provides a potential approach to reduce the total commuting time in a neutral-fiscal way. Based on the HOV lane management model given in Section 2.3, we can combine the TCS into the HOV lane management problem. That is, the HOV lane management can be formulated as Bilevel Programming 5-10 when TCS applies.

$$
\begin{aligned}
& \min _{\rho, \kappa_{1}, \kappa_{2}}\left[f_{T}\left(\frac{1}{2} n, \rho C\right)+D\right] n+ \\
& +f_{T}((N-n),(1-\rho) C)(N-n)
\end{aligned}
$$

subject to Constraint 2 and

$\left(\kappa_{1}-1\right)\left(\kappa_{2}-1\right) \leq 0$

$\kappa_{1} \geq 0$

$\kappa_{2} \geq 0$

where $n$ solves the following deterministic equilibrium problem

$\min _{n} \int_{0}^{n}\left[f_{T}\left(\frac{x}{2}, \rho C\right)+D\right] d x+\int_{0}^{N-n} f_{T}(x,(1-\rho) C) d x$

subject to Constraint 4 and

$\kappa_{1}(N-n)+\kappa_{2} n \leq N$

Bilevel Programming 5-10 explains how to combine HOV lanes and TCSs to relieve traffic congestion. The number of commuters who choose HOV lanes under TCS is determined by the lower-level programming. Comparing to the lower-level programming of Bilevel Programming 1-4, Constraint 10 is added. This constraint renders that the solution of this lower-level programming is under equilibrium with a TCS. Moreover, the Lagrangian multiplier of Constraint 10 is 
exactly the price of credit. The objective function of the upper-level programming of Bilevel Programming 5-10 is the same as that of Bilevel Programming 1-4, and the credit tolls $\kappa_{1}$ and $\kappa_{2}$ are added into decision variables. Constraints 6-8 of the upper-level programming ensure that $\kappa_{1}$ and $\kappa_{2}$ are reasonable. Noting that $\kappa_{1}$ and $\kappa_{2}$ are non-negative and cannot be set too high simultaneously, i.e. $\kappa_{1}>1$ and $\kappa_{2}>1$ (if so, some commuters will not have enough credits to trade which is not in agreement with the assumption of the proposed model). Moreover, it will be of no effect if these two values are set too small at the same time, i.e. $0<\kappa_{1}<1$ and $0<\kappa_{2}<1$, which means every commuter has enough credits to choose between driving alone or carpooling. These two cases of credit settings are integrated into Constraint 6.

\section{ALGORITHM DESIGN}

Genetic Algorithms (GA) is applied to solve the proposed two bilevel programming and the interior point algorithm [17] is used to solve the lower-level programming. GA, as the wide-used heuristic algorithm, has features of global convergence which fits for solving the proposed bilevel programming (see Remark 4).

Remark 4. At first, we plan to apply a classical numerical algorithm for solving both levels of bi-level programming. However, the upper-level programming cannot guarantee one local optimal solution in the feasible area. Different initial values result in different local optimal solutions. In order to ensure that the optimal solution is achieved at every point of the solving process, GA is used in our study.

The algorithm framework is given below. Firstly, several chromosomes are randomly generated to form the initial population. Secondly, the mutation operator is applied to a part of chromosomes with mutation probability. Thirdly, the crossover operator is applied to some chromosomes with crossover probability. Fourthly, the new population is generated according to the selection of probability, which is calculated by the fitness function of the value of chromosome decoded under the coding scheme. Finally, the new population becomes the initial population of the next round till the algorithm stops.

\section{Coding scheme}

In the proposed GA, each chromosome $\Xi$ is composed of random variables and subjects to the uniform distribution between 0 and 1 . A special coding scheme is designed by mapping from the space of chromosomes to the feasible set of the programming model $5-10$. The setting of the coding scheme is based on the following Function 11.

$$
\rho=\xi_{1}, \kappa_{1}=\frac{\xi_{2}}{\xi_{3}}, \kappa_{2}=\frac{1-\xi_{2}}{1-\xi_{3}}
$$

where $\xi_{1}, \xi_{2}$ and $\xi_{3}$ are three random variables subjecting to the uniform distribution between 0 and 1 . Each chromosome consists of $\xi_{1}, \xi_{2}$ and $\xi_{3}$ that is, $\Xi=\left(\xi_{1}, \xi_{2}, \xi_{3}\right)$.

This coding scheme ensures that there is a oneto-one correspondence between the chromosome and the feasible solution, except for boundary points. The generated random solutions traverse the whole feasible region effectively while the verification process can be omitted to reduce the calculation time. The relevant properties of the coding scheme are proved in the Appendix.

\section{Mutation operator}

A classic mutation operator is used in the genetic algorithm.

$\Xi_{i}^{\prime}=\left(1-\varepsilon^{2}\right) \Xi_{i}+\varepsilon^{2} \Xi^{*}$

where $\Xi^{*}$ is a new random chromosome, $\Xi_{i}$ is the chosen chromosome of mutation, $\Xi_{i}^{\prime}$ is the new chromosome after mutation and $\varepsilon$ is random variable subject to a uniform distribution between 0 and 1 .

\section{Crossover operator}

The crossover operator is applied in the genetic algorithm, as shown in Equations 13, 14.

$\Xi_{i}^{\prime}=\varepsilon^{2} \Xi_{i}+\left(1-\varepsilon^{2}\right) \Xi_{j}$

$\Xi_{j}^{\prime}=\left(1-\varepsilon^{2}\right) \Xi_{i}+\varepsilon^{2} \Xi_{j}$

where $\Xi_{i}$ and $\Xi_{j}$ are chosen chromosomes of crossover, $\Xi_{i}^{\prime}$ and $\Xi_{j}^{\prime}$ are the new chromosomes after crossover and $\varepsilon$ is a random variable subject to a uniform distribution between 0 and 1 .

\section{Selection operator}

The selection operator is given in the following equations:

$$
\begin{aligned}
\Phi_{\text {Max }} & =\max _{i}, \Phi_{i}, \Phi_{\text {Min }}=\min _{i}, \Phi_{i}, \Phi_{i}^{+}= \\
& =\left(\frac{\Phi_{i}-\Phi_{\text {Min }}}{\Phi_{\text {Max }}-\Phi_{\text {Min }}}\right)^{2}, \Phi_{i}^{*}=\frac{\Phi_{i}^{+}}{\sum_{k} \Phi_{k}^{+}}, \quad i \in I
\end{aligned}
$$

where $I$ is the index set of chromosomes, $\Phi_{i}$ is the value of upper-level objective function under the decision variables which are determined by the corresponding chromosome $\Xi_{i} ; \Phi_{\text {Max }}$ and $\Phi_{\text {Min }}$ are maximum and minimum of all values and $\Phi_{i}^{*}$ is the roulette probability of $i$-th chromosome. In the process of calculating $\Phi_{i}$, the lower-level programming model can be solved by an interior point algorithm [17].

\section{NUMERICAL EXAMPLES}

We first introduce the congestion function and parameters used in this study. As shown in Equation 16, the BPR function for its originators at the US Bureau of Public Roads is adopted, which has wide use in 
urban transportation planning practice. The congestion function is used to approach the delay caused by over-crowding.

$$
f_{T}(c, n)=30 \cdot\left(1+0.15\left(\frac{n}{c}\right)^{4}\right)
$$

where the free-flow travel time is 30 minutes.

The travel time is a free-flow travel time if no commuter chooses the current mode and increases with the number of commuters. Different from the original BPR function, which estimates the travel time of a certain link based on the flow and capacity (vehicles per hour) of the link, the congestion function is used here to estimate the travel time of commuters, based on the number of commuters and the capacity of a type of lane.

Other model parameters are listed in Table 2.

Table 2 - Value and unit of model parameters

\begin{tabular}{|c|c|c|}
\hline Notations & Value & Unit \\
\hline \hline N & 10,000 & Commuter \\
\hline C & 6,000 & Commuter \\
\hline D & 30 & Minute \\
\hline
\end{tabular}

Parameters used in the genetic algorithm are listed in Table 3.

Table 3 - Value of algorithm parameters

\begin{tabular}{|l|c|}
\hline \multicolumn{1}{|c|}{ Parameters } & Value \\
\hline \hline Number of generation & 64 \\
\hline Number of chromosome & 32 \\
\hline Probability of crossover & 0.4 \\
\hline Probability of mutation & 0.15 \\
\hline
\end{tabular}

\subsection{Investigation of fixed TCS under a fixed HOV capacity ratio}

At first, the effect of a fixed TCS is investigated. Considering the case with the HOV capacity ratio, $\rho=0.33$, and the credit tolls, $\kappa_{1}=1.2$ and $\kappa_{2}=0.8$.

Table 4 - Impact of fixed TCS under fixed HOV capacity ratio

\begin{tabular}{||l|c|c||}
\hline \multicolumn{1}{|c|}{ Case } & Without TCS & With TCS \\
\hline \hline Total commuting time [min] & 624,649 & 557,933 \\
\hline $\begin{array}{l}\text { Number of LOV commuters } \\
\text { Number of HOV commuters }\end{array}$ & $\begin{array}{l}6,490 \\
3,510\end{array}$ & $\begin{array}{l}5,000 \\
5,000\end{array}$ \\
\hline $\begin{array}{l}\text { Commuting time of } \\
\text { LOV commuters [min] }\end{array}$ & 62 & 41 \\
\hline $\begin{array}{l}\text { Commuting time } \\
\text { of HOV commuters [min] }\end{array}$ & 62 & 70 \\
\hline
\end{tabular}

When the HOV capacity ratio is fixed under the user equilibrium, Table 4 demonstrates the changes of the total commuting time, the number of LOV commuters and HOV commuters, the commuting time of LOV commuters and HOV commuters with and without fixed TCS. With the credit tolls $\left(\kappa_{1}=1.2\right.$ and $\kappa_{2}=0.8$.), the number of HOV commuters increases and the number of LOV commuters decreases. The changes of the two group numbers also bring a decrease of the commuting time of LOV commuters (from 62 to 41) and a slight increase of the commuting time of HOV commuters (from 62 to 70 ), and the total commuting time decreases (from 624,649 to 557,933 ). That is, the presences of TCSs with HOV lanes mitigates traffic congestion.

\subsection{Investigation of optimal TCS}

In order to describe the optimal TCS clearly, an index of TCSs, the credit toll proportion (CTP) $\Pi$, is defined first. The CTP is the proportion of the number of credits that LOV commuters need to purchase and the number of credits that HOV commuters can sell, which is formulated as

$\Pi=\frac{\kappa_{1}-1}{1-\kappa_{2}}$

According to 128 runs of GA, the optimal credit tolls are illustrated in Figure 1.

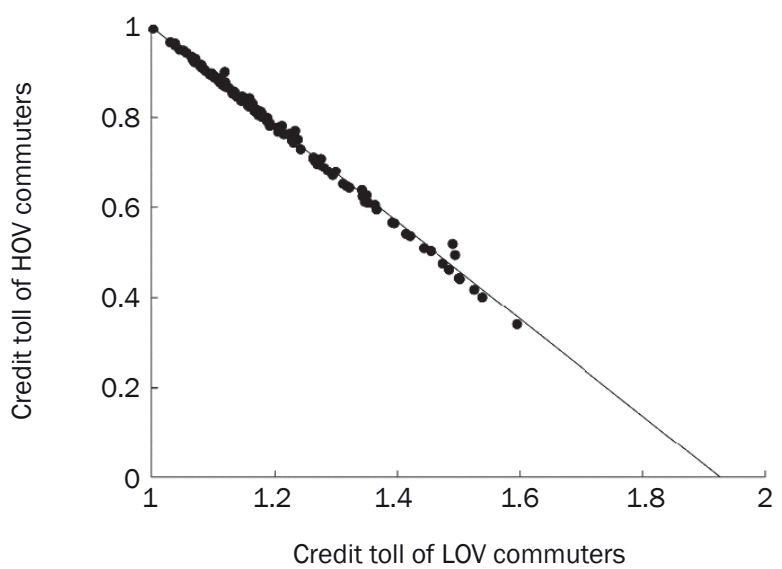

Figure 1 - Comparison of pairs of credit tolls

As shown in Figure 1, the pairs of optimal credit tolls are not the same but almost in a line in this coordinate. It means that the CTPs of these optimal TCSs solved by GA are almost equal ( $\rho=0.9264)$.

The CTP of TCSs provides an effective index of the proposed model. To continue the investigation, a new numerical example according to the optimal solutions based on the 128 runs is presented. HOV capacity ratio is set as the mean of optimal HOV capacity ratios $(\rho=0.35)$ and a new TCS $\left(\kappa_{1}=1.4632\right.$ and $\left.\kappa_{2}=0.5\right)$ satisfying the optimal CTP $(\Pi=0.9264)$. The solution is listed in Table 5. 
Table 5 - Commuting time and number of commuters with the optimal TCS

\begin{tabular}{||l|c|c|c||}
\hline \multicolumn{1}{|c|}{ Mode } & LOV & HOV & Mean \\
\hline \hline Commuting time [min] & 43.91 & 67.96 & 55.47 \\
\hline $\begin{array}{l}\text { Difference of mean } \\
\text { commuting time [min] }\end{array}$ & -11.56 & 12.48 & - \\
\hline Number of commuters & 5,191 & 4,809 & - \\
\hline
\end{tabular}

As shown in Table 5 each LOV commuter will spend 43.91 minutes for commuting. It is 11.56 minutes less than the mean commuting time but needs to purchase an extra number of credits $(0.4632$, the initial number of credits that every commuter acquires is 1) from the market. On account of the user equilibrium under TCS, both types of commuters should spend the same commuting cost (55.47 min) which is equal to the sum of the commuting time (43.91 $\mathrm{min}$ ) and the credit cost (11.56 $\mathrm{min})$. It results in that the unit price (measured by minutes in the proposed model) of credit is 24.97 $(11.56 / 0.4632)$ minutes. On the contrary, the commuting time of HOV commuters is more than the average commuting time but they can be compensated by selling an extra number of credits. Therefore, by applying the given TCS, the two types of commuters attain the new user equilibrium by buying and selling credits whilst the total commuting time decreases.

Furthermore, because the number of commuters multiplied by the credit toll for both travel modes is equal according to the conservation of credits, the proportion of the number of HOV commuters and LOV commuters $0.9264(5191 / 4809)$ is equal to CTP.

\subsection{Comparative analysis}

Two parameters in the proposed models, the carpool organization time and the number of commuters, are investigated in this section.

\subsubsection{Carpool organization time}

Figures 2 and 3 illustrate the changes of the total commuting time and the optimal HOV capacity ratio with respect to the carpool organization time with and without the presence of TCSs, respectively. Figure 4 shows the CTP at different carpool organization times.

As shown in Figure 2, whether with the presence of TCS or not, the total commuting time increases with the carpool organization time. The total commuting time is always larger with TCSs. Without TCSs, the total commuting time grows quickly with the low carpool organization time (between 10 to 35), and then it tends to be stable at $660,000 \mathrm{~min}$. Comparing the total commuting time without and with TCSs, the gaps increase firstly and then decrease with the increase of the carpool organization time, and the almost maximum gap appears when the carpool organization time is between 30 to 35. Correspondingly, as shown in Figure 3,

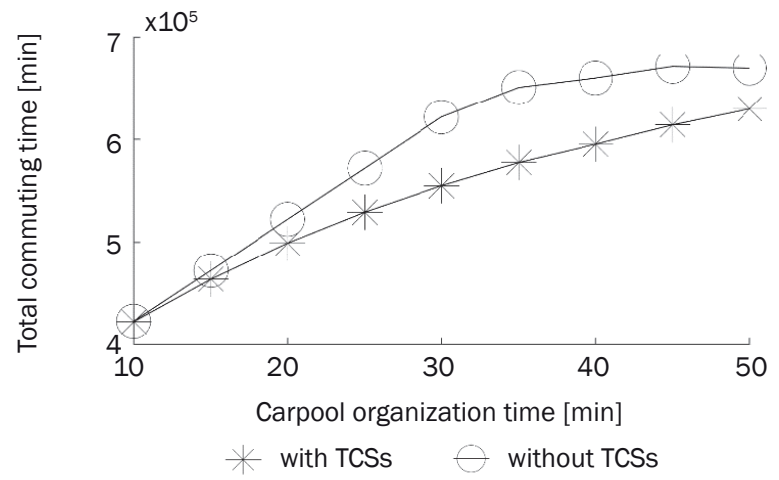

Figure 2 - Total commuting time by different carpool organization times, with and without TCSs

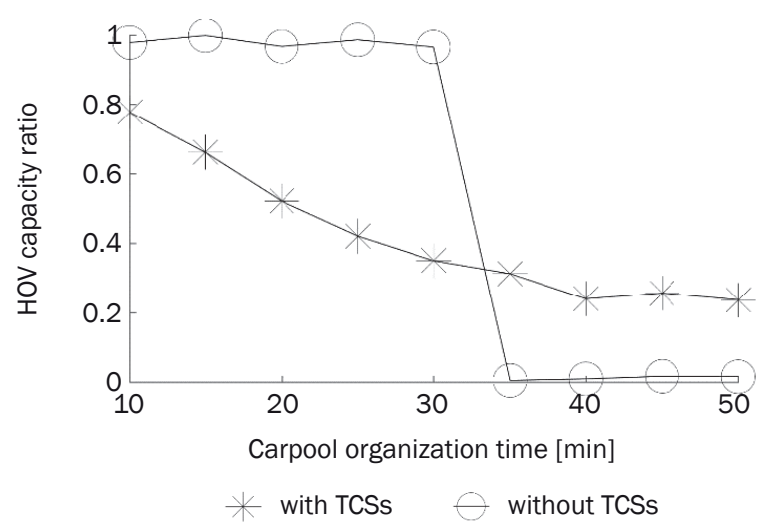

Figure 3 - HOV capacity ratio by different carpool organization times, with and without TCSs

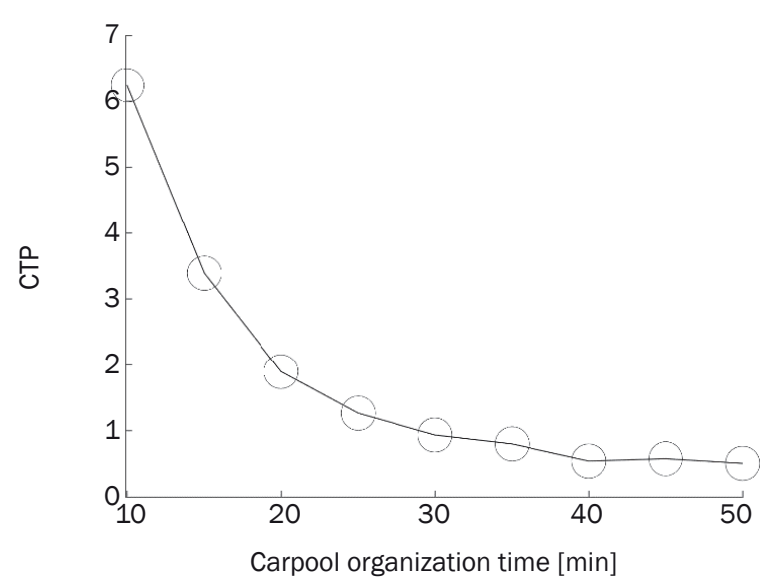

Figure 4 - Changes of CTPs by different carpool organization times, with TCSs

without TCSs, the HOV capacity ratio has a sharp drop from 1 to 0 when the carpool time is between 30 to 35 , which differs from the change of HOV capacity ratio at the presence of TCSs, where the HOV capacity ratio decreases gradually. Furthermore, in the presence of TCSs, the CTP decreases with the increase of the carpool organization time in Figure 4. It also means that the number of HOV commuters also decreases with the growth of the carpool organization time. 
Without TCSs, both LOV commuters and HOV commuters choose their own travel mode depending on their own commuting time. If the carpool organization time is short, the HOV mode is more time-efficient. In consequence, the highway authority tends to set all lanes as HOV lanes to minimize the total commuting time. With the growth of the carpool organization time, there is a threshold between 30 to 35 in this numerical example, and the HOV mode is no longer the prior choice for commuters when the carpool organization time is over the threshold. In this case, no HOV lanes will be further set, and the maximal total commuting time of this numerical example arrives. Since the TCS is not implemented to affect commuters' travel choices, this all-or-nothing situation of HOV lanes is inevitable. Once the threshold value is below the carpool organization time, the highway authority can only cancel all HOV lanes to achieve the minimal total commuting time. Otherwise, if some HOV lanes are retained, the total commuting time will be higher. Further, in the presence of TCSs, the changes of the HOV capacity ratio is gradual and therefore the traffic condition is always better because of a significant decrease and a slight increase of the commuting time in GP lanes and HOV lanes, respectively, and the number of HOV commuters decreases gradually. Furthermore, when the CTP is about 1, which means the numbers of commuters of both modes are equal, most effect can be achieved with TCSs; otherwise, TCS will have less effects in improving traffic conditions whatever mode dominates.

\subsubsection{The number of commuters}

With respect to the effect of the total number of commuters to traffic conditions, Figures 5 and 6 illustrate the changes of the total commuting time and the optimal HOV capacity ratio with the growth of the number of commuters with and without the presence of TCSs, and Figure 7 shows the changes of CTP with the growth of the number of commuters in the presence of TCSs.

As shown in Figure 5, whether with the presence of TCSs or not, the total commuting time increases with the growth of the number of commuters, and the total commuting time without the presence of TCSs is always no less than the corresponding total commuting time with the presence of TCSs. The HOV capacity ratios with respect to the number of commuters are illustrated in Figure 6 and CTP with respect to the number of commuters is shown in Figure 7, which demonstrates opposite tendency with the corresponding case with respect to the carpool organization time, as presented in Figures 3 and 4. According to Figure 6, without the presence of TCSs, there is a threshold when the number of commuters is between 9,000 and 10,000, where CTP changes from 0 to 1 . However, with

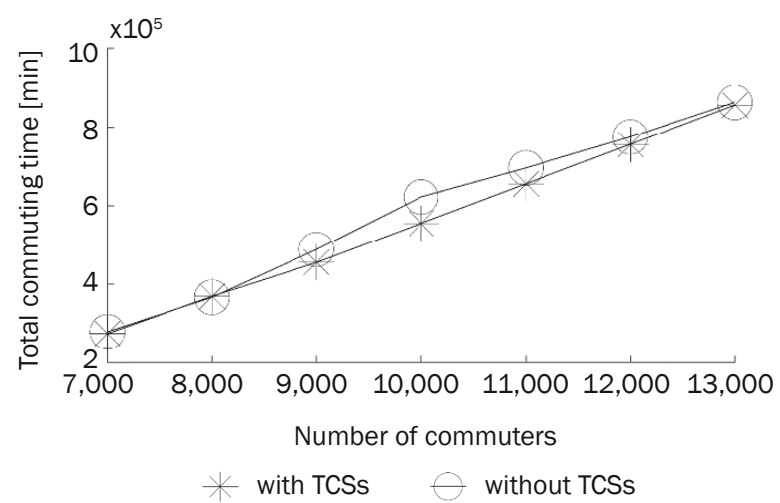

Figure 5 - Total commuting time by different commuters, with and without TCS

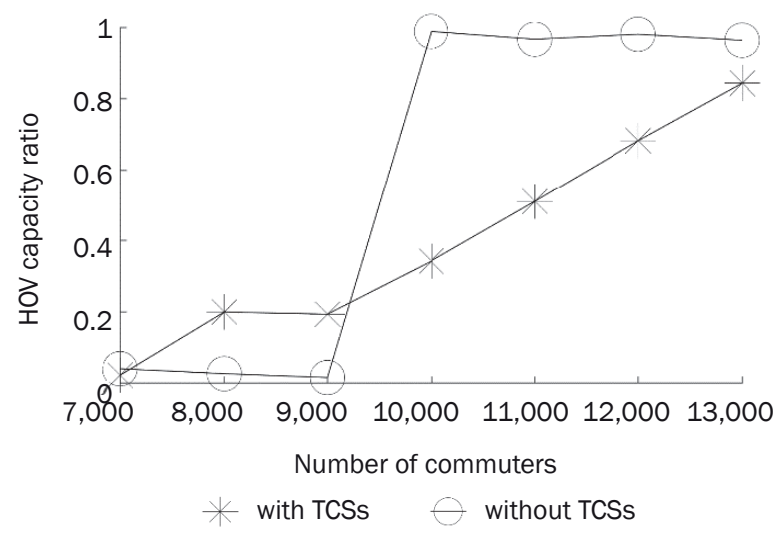

Figure 6 - HOV capacity ratio by different commuters, with and without TCS

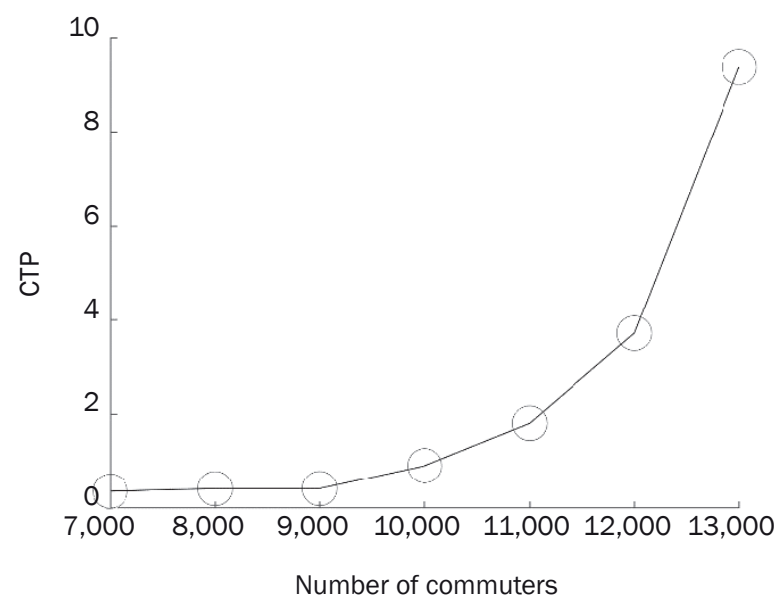

Figure 7 - Changes of CTPs under different numbers of commuters

the presence of TCSs, CTP grows gradually from 0 to 1 while the number of commuters increases from 9,000 to 10,000 . Figure 7 shows that the CTP increases with the growth of the number of commuters, which means that the ratio of the number of HOV commuters with respect to the total number of commuters increases with the growth of the total number of commuters. 
Without the presence of TCSs, as shown in Figure 5, there is a threshold of the total commuting time with respect to the total number of commuters. When the total number of commuters is less than the threshold between 9,000 and 10,000, the highway authority does not need to set any HOV lanes and the traffic condition is optimal. However, when the number of commuters exceeds the threshold, all lanes should be set as HOV lanes; otherwise, the total commuting time does not achieve the minimum. The reason of this phenomenon is that only the presence of HOV lanes cannot guarantee the minimal commuting time, which is similar to the analysis in the case with respect to the carpool organization time in Section 4.2.1. However, different from the case in Section 4.2.1, there is no maximal total commuting time because a larger number of commuters always results in worse traffic conditions.

With the presence of TCSs, the highway authority enables any number of commuters to choose LOV mode or HOV mode according to the setting of TCS which is able to lead to a decrease of the total commuting time. Furthermore, as the total number of commuters increases, the highway authority could add HOV lanes and increase the CTP so that more commuters would choose HOV mode in order to decrease the total commuting time.

\section{CONCLUSION}

In this paper, a bilevel programming approach is presented to model the HOV lane management problem with/without the TCS policy. Considering the complexity of solving the proposed bilevel programming model, the GA is applied to search for the optimal solutions. According to the numerical examples, the following conclusions have been made: (1) Comparing the HOV lane management with/without the TCS policy, the combination of HOV lanes and TCSs can obviously reduce the total commuting time. (2) The proposed bilevel programming approach provides an analytical method to deal with the HOV lane management problem. The decision variables of the bilevel programming model, i.e. the optimal HOV capacity ratio and the optimal LOV and HOV credit tolls, can be calculated by the proposed GA.

The HOV lane management problem studied in this paper investigates the reasonable setting of HOV lanes, and encourages commuters to use HOV lanes with TCS application. This study presents a series of issues for further studies, alongside some other key issues, e.g., the HOV lane management problem under the elastic demand case, consideration of heterogeneous commuters with different carpool organization times, and further application-related issues of TCSs in the presence of HOV lanes.

\section{ACKNOWLEDGEMENTS}

The work described in this paper was jointly supported by the National Natural Science Foundation of China (71422010, 71621001), and the State Key Laboratory of Rail Traffic Control and Safety (Contract No. RCS2017ZZ001), Beijing Jiaotong University.

\section{Appendix: Properties of the Coding Scheme 11}

Before we further prove the properties of the genetic algorithm coding scheme, it should be noted that the boundary value of constraints in the programming problem has not been discussed. (Conditions 2, 6, 7, 8 are revised as $18,19,20,21$.)

$0<\rho<1$

$\left(\kappa_{1}-1\right)\left(\kappa_{2}-1\right)<0$

$\kappa_{1}>0$

$\kappa_{2}>0$

Proposition 1. For any $\xi_{1}, \xi_{2}, \xi_{3} \in(0,1)$ and $\xi_{2} \neq \xi_{3}$, if $\rho, \kappa_{1}, \kappa_{2}$ satisfy $11, \rho, \kappa_{1}, \kappa_{2}$ will satisfy $2,6,7,8$. Proof:

1) $\rho=\xi_{1} \in(0,1)$

2) Since $\xi_{2}, \xi_{3} \in(0,1), \xi_{2} \neq \xi_{3}$ and 11 , we have $\left(\kappa_{1}-1\right)\left(\kappa_{2}-1\right)=\frac{\xi_{3}-\xi_{2}}{\xi_{3}} \frac{\xi_{2}-\xi_{3}}{1-\xi_{3}}=\frac{-\left(\xi_{3}-\xi_{2}\right)^{2}}{\xi_{3}\left(1-\xi_{3}\right)}<0$

3) Since $\xi_{2}, \xi_{3} \in(0,1)$, we have $\kappa_{1}=\frac{\xi_{2}}{\xi_{3}}>0$.

4) Since $\xi_{2}, \xi_{3} \in(0,1)$, we have $\kappa_{2}=\frac{1-\xi_{2}}{1-\xi_{3}}>0$.

Proposition 2. For any $\rho, \kappa_{1}, \kappa_{2}$ satisfying $18,19,20,21$,

there exist $\xi_{1}, \xi_{2,} \xi_{3} \in(0,1)$ and $\xi_{2} \neq \xi_{3}$ satisfy 11 .

Proof:

Let $\xi_{1}, \xi_{2}, \xi_{3}$ satisfy $\xi_{1}=\rho, \xi_{2}=\frac{\kappa_{1}}{\kappa_{2}-\kappa_{1}}\left(\kappa_{2}-1\right)$, $\xi_{3}=\frac{1}{\kappa_{2}-\kappa_{1}}\left(\kappa_{2}-1\right)$. If $\kappa_{2}>1$, according to 6 , we have $\kappa_{2}>1>\kappa_{1}$ and hence $\kappa_{2}-\kappa_{1}>\kappa_{2}-1>0$ and $\kappa_{1}<1$.

Therefore, $\xi_{1}=\rho \in(0,1), \xi_{2}=\frac{\kappa_{1}}{\kappa_{2}-\kappa_{1}}\left(\kappa_{2}-1\right) \in(0,1)$, $\xi_{3}=\frac{1}{\kappa_{2}-\kappa_{1}}\left(\kappa_{2}-1\right) \in(0,1)$. We also have $\kappa_{1}=\frac{\xi_{2}}{\xi_{3}}, \kappa_{2}=\frac{1-\xi_{2}}{1-\xi_{3}}$.

Proposition 3. For any, $\xi_{1}, \xi_{2}, \xi_{3} \in(0,1), \xi_{2} \neq \xi_{3}$ and $\xi_{1}^{\prime}, \xi_{2}^{\prime}, \xi_{3}^{\prime} \in(0,1), \xi_{2} \neq \xi_{3}$ satisfy

$\left(\xi_{1}, \xi_{2}, \xi_{3}\right) \neq\left(\xi_{1}^{\prime}, \xi_{2}^{\prime}, \xi_{3}^{\prime}\right)$, if $\rho=\xi_{1}, \kappa_{1}=\frac{\xi_{2}}{\xi_{3}}$,

$\kappa_{2}=\frac{1-\xi_{2}}{1-\xi_{3}}, \rho^{\prime}=\xi_{1}^{\prime}, \kappa_{1}^{\prime}=\frac{\xi_{2}^{\prime}}{\xi_{3}^{\prime}}, \kappa_{2}^{\prime}=\frac{1-\xi_{2}^{\prime}}{1-\xi_{3}^{\prime}}$,

$\left(\rho, \kappa_{1}, \kappa_{2}\right) \neq\left(\rho, \kappa_{1}^{\prime}, \kappa_{2}^{\prime}\right)$ is satisfied. 
Proof:

Assume $\left(\rho, \kappa_{1}, \kappa_{2}\right)=\left(\rho^{\prime}, \kappa_{1}^{\prime}, \kappa_{2}^{\prime}\right)$. Then, we have $\xi_{1}=\rho=\rho^{\prime}=\xi_{1}^{\prime}$

$\xi_{2}=\frac{\kappa_{1}}{\kappa_{2}-\kappa_{1}}\left(\kappa_{2}-1\right)=\frac{\kappa_{1}^{\prime}}{\kappa_{2}^{\prime}-\kappa_{1}^{\prime}}\left(\kappa_{2}^{\prime}-1\right)=\xi_{2}^{\prime}$

$\xi_{3}=\frac{1}{\kappa_{2}-\kappa_{1}}\left(\kappa_{2}-1\right)=\frac{1}{\kappa_{2}^{\prime}-\kappa_{1}^{\prime}}\left(\kappa_{2}^{\prime}-1\right)=\xi_{3}^{\prime}$. There-

fore, we have $\left(\xi_{1}, \xi_{2}, \xi_{3}\right)=\left(\xi_{1}^{\prime}, \xi_{2}^{\prime}, \xi_{3}^{\prime}\right)$. However, it is contrary to the condition. This completes the proof.

\section{蔵广智1}

gzzang@bjtu.edu.cn

徐猛（通讯作者） 1

mengxu@bjtu. edu.cn

高自友 ${ }^{1}$

zygao@bjtu. edu.cn

1 轨道交通控制与安全国家重点实验室,

北京交通大学, 北京, 100044, 中国

\section{利用高占有率车道和可交易出行票进行交通拥堵管} 理：基于双层规划方法

\section{摘要}

高占有率车道是一种鼓励更多的人使用高占有率出行 方式的交通管理手段。该手段旨在促进更多的人使用同一 条道路出行，目前已经被应用于缓解日益增长的交通拥 堵。但是，在该手段的执行过程中，由于高占有率车道没 有合理的设置，并未达到预期的效果。在本文中，我们引 入可交易出行票以提升高占有率车道的管理效果, 并同时 研究为高占有率车道设置最优的通行能力以匹配可交易出 行票。通过利用双层规划建模方法，上层规划表示高速管 理部门的决策，而下层遵循固定需求下通勤者的用户均 衡。我们进一步研究了可交易出行票的潜在影响, 提出了 一个修正的遗传算法求解本文提出的双层规划模型。数值 算例显示结合高占有率车道和可交易通行票两种交通管理 策略可以明显的缓解交通拥堵。

\section{关键词}

高占有率车道; 车道管理; 可交易出行票; 交通需求管 理; 均衡

\section{REFERENCES}

[1] Neudorff LG, Randall JE, Reiss R, Gordon R. Freeway management and operations handbook. New York: Federal Highway Administration; 2006.

[2] Bing X. What caused the difficulty in designating HOV lanes? China Youth News. Aug 102016.

[3] Shewmake S. Can Carpooling Clear the Road and Clean the Air?: Evidence from the Literature on the Impact of HOV Lanes on VMT and Air Pollution. Journal of Planning Literature. 2012;27(4): 363-74.

[4] Yang $\mathrm{H}$. When should carpool lanes be introduced in a multi-lane highway? Journal of Advanced Transportation. 1998;32(2): 242-52.

[5] Yang $\mathrm{H}$, Huang $\mathrm{H}-\mathrm{J}$. Carpooling and congestion pricing in a multilane highway with high-occupancy-vehicle lanes. Transportation Research Part A: Policy and Practice. 1999;33(2): 139-55.

[6] Konishi H, Mun S-I. Carpooling and congestion pricing: HOV and HOT lanes. Regional Science and Urban Economics. 2010;40(4): 173-86.

[7] Small KA, Yan J. The Value of Value Pricing of Roads: Second-Best Pricing and Product Differentiation. Journal of Urban Economics. 2001;49(2): 310-36.

[8] Verhoef ET, Small KA. Product Differentiation on Roads. Journal of Transport Economics and Policy (JTEP). 2004;38(1): 127-56.

[9] Fuhs C, Obenberger J. Development of High-Occupancy Vehicle Facilities: Review of National Trends. Transportation Research Record. 2002;1781: 1-9.

[10] Furuhata M, Dessouky M, Ordonez F, Brunet M-E, Wang X, Koenig S. Ridesharing: The state-of-the-art and future directions. Transportation Research Part B: Methodological. 2013;57: 28-46.

[11] De Corla-Souza P. Fair highway networks: a new approach to eliminate congestion on metropolitan freeways. Public Works Management and Policy. 2005;9(3): 196-205.

[12] Fan W, Jiang X, Erdogan S, Sun Y. Modeling and evaluating FAIR highway performance and policy options. Transport Policy. 2016;48: 156-168.

[13] Grant-Muller S, Xu M. The Role of Tradable Credit Schemes in Road Traffic Congestion Management. Transport Reviews. 2014;34(2): 128-49.

[14] Yang H, Wang X. Managing network mobility with tradable credits. Transportation Research Part B: Methodological. 2011;45(3): 580-94.

[15] Fan W, Jiang $X$. Tradable mobility permits in roadway capacity allocation: Review and appraisal. Transport Policy. 2013;30: 132-42.

[16] Sheffi Y (ed.). Urban Transportation Networks: Equilibrium Analysis with Mathematical Programming Methods. Englewood Cliffs, N.J.: Prentice-Hall; 1985.

[17] Byrd RH, Hribar ME, Nocedal J. An Interior Point Algorithm for Large-Scale Nonlinear Programming. SIAM J on Optimization. 1999;9(4): 877-900. 